PROCEEDINGS OF THE

AMERICAN MATHEMATICAL SOCIETY

Volume 138, Number 7, July 2010, Pages 2505-2511

S 0002-9939(10)10278-0

Article electronically published on February 18, 2010

\title{
APPROXIMATION BY STRONGLY ANNULAR SOLUTIONS OF FUNCTIONAL EQUATIONS
}

\author{
R. DAQUILA
}

(Communicated by Walter Van Assche)

\begin{abstract}
A major result of this paper is that the set of all functions $g(z)$ such that $g$ is strongly annular and is a solution of a Mahler type of functional equation given by $g(z)=q(z) g\left(z^{p}\right)$ where $p \geq 2$ is an integer and $q$ is a polynomial with $q(0)=1$ is a dense first category set in the set of all holomorphic functions on the open unit disk with the topology of almost uniform convergence. A second result is that strongly annular solutions of these types of functional equations are dense in the space of holomorphic functions with Maclaurin coefficients of \pm 1 with the same topology.
\end{abstract}

\section{INTRODUCTION}

A function $g$ holomorphic on the open unit disk is said to be strongly annular if there exists a strictly increasing sequence of positive real numbers $\left\{r_{n}\right\}$ converging to 1 such that the minimum modulus of $g$ goes to infinity on circles centered at the origin of radius $r_{n}$. Bonar 2] has summarized much of the development of the subject of annular functions. A non-constant holomorphic function $g$ is a solution to the Mahler type of functional equation if there is an integer $p \geq 2$ and a polynomial $q$ with $q(0)=1$ such that $g$ satisfies the functional equation

$$
g(z)=q(z) g\left(z^{p}\right) .
$$

It has been shown by Daquila $[5$, that if

$$
q(0)=1 \text { and } \liminf _{r \rightarrow 1^{-}} \min _{|z|=r}|q(z)|>1,
$$

then the unique solution $g$ of (1.1) for which $g(0)=1$ is a strongly annular function.

The focus of this paper is to show that any function holomorphic on the open unit disk can be approximated by a solution to a Mahler type of functional equation (1.1). We denote all the solutions to the functional equation (1.1) as $M$. The set $M$ can be characterized as the set of all non-constant holomorphic functions $g$ on the unit disk such that, for some integer $p, p \geq 2$, the meromorphic function $g(z) / g\left(z^{p}\right)$ is actually a polynomial whose value at the origin is 1 .

In the second section of this paper we show that the set of all functions $g$ which are strongly annular and are in the set $M$ form a dense first category set in the set of all functions holomorphic on the open unit disk with the topology of almost

Received by the editors July 31, 2009, and, in revised form, October 20, 2009, and November 5, 2009.

2010 Mathematics Subject Classification. Primary 30D10, 30B30, 30E10, 41A30.

(C)2010 American Mathematical Society

Reverts to public domain 28 years from publication 
uniform convergence. This result is not intuitively obvious based on the bound found by Becker [1] in estimating the rate of growth of polynomial combinations of solutions to this functional equation. It has been proven by Bonar and Carroll [3] that annular functions form a residual set with this same topology.

In the third section of this paper we show that if we consider only holomorphic functions whose Maclaurin coefficients are \pm 1 , then the set of strongly annular solutions with coefficients of \pm 1 that are solutions to the set of functional equations is dense in the topology of almost uniform convergence. The set of strongly annular functions with coefficients of \pm 1 has been studied by Howell [7, who was able to show that this set of functions forms a residual set. We note that the set of solutions to the functional equations (1.1) where $q$ is a polynomial with coefficients \pm 1 and $p \geq 2$ is an integer is countable and obviously of first category.

\section{HOLOMORPHIC FUNCTIONS}

The solution of a functional equation of type (1.1) for a given $q$ and $p$ is uniquely determined up to a multiplicative complex constant. The focus of this section is approximating a holomorphic function by a solution of equation (1.1) that is strongly annular. A version of Runge's theorem is stated as follows.

Theorem 2.1 (Runge's theorem). The polynomials with complex coefficients are dense in the set of all functions holomorphic on the open unit disk with the topology of almost uniform convergence. (See Conway [4] p. 198.)

To show that the set of functions $M$ is dense it is enough to show that the set $M$ is dense in the set of polynomials described above. It is important to note that if a stongly annular function is multiplied by a non-zero constant the resulting function is strongly annular.

Lemma 2.2. The set of functions $\{c q(z): c \in \mathbb{C}, c \neq 0, q$ is a polynomial satifying (1.2) $\}$ is dense in the set of all functions holomorphic in the open unit disk with the topology of almost uniform convergence.

Proof. Since polynomials are dense in this topology by Runge's theorem, it suffices to prove that the set of functions described in Lemma 2.2 is dense in the set of polynomials.

Let $K$ be a compact subset of the open unit disk, and let $\epsilon>0$ be given. Let $h(z)$ be an arbitrary polynomial with coefficients $a_{j}$ :

$$
h(z)=\sum_{j=0}^{n} a_{j} z^{j} .
$$

Choose a number $b_{0} \neq 0$ such that $\left|a_{0}-b_{0}\right|<\epsilon / 2$. Set $b_{i}=a_{i}$ for $i=1,2, \ldots, n$. Define the numbers $c_{j}=b_{j} / b_{0}$ for $j=0,1,2, \ldots, n$. A number $A \in \mathbb{C}$ can be chosen such that the following inequality is satisfied:

$$
|A|-\sum_{j=0}^{n}\left|c_{j}\right|>1 .
$$

Choose a positive integer $m>n=\operatorname{deg} h$ such that $\left|b_{0}\right||A||z|^{m}<\epsilon / 2$ on $K$. The polynomials $b(z)$ and $q(z)$ are defined below such that the coefficients of $h(z)$ are 
equal to the coefficients of $b(z)$ (except for the constant term) and the coefficients of the polynomial $b_{0} q(z)$ are equal to $b(z)$ (except for the $z^{m}$ term):

$$
b(z)=\sum_{j=0}^{n} b_{j} z^{j} \text { and } q(z)=\sum_{j=0}^{n} c_{j} z^{j}+A z^{m} .
$$

The polynomial $q(z)$ has been constructed so that it satisfies the conditions of (1.2). It is clear from the choice of coefficients that the value of $q(z)$ at the origin is 1 . The minimum modulus of the polynomial $q$ on the boundary of the unit disk can be estimated using inequality (2.1):

$$
\min _{|z|=1}|q(z)| \geq|A|-\sum_{j=0}^{n}\left|c_{j}\right|>1 .
$$

The polynomial $b_{0} q(z)$ approximates the function $h(z)$ on the compact set $K$. That is, for all $z$ in $K$ we have the following inequality:

$$
\begin{aligned}
\left|h(z)-b_{0} q(z)\right| & \leq|h(z)-b(z)|+\left|b(z)-b_{0} q(z)\right| \\
& =\left|a_{0}-b_{0}\right|+\left|b_{0}\right||A||z|^{m}<\epsilon .
\end{aligned}
$$

Thus we have shown, for any polynomial $h$ and compact subset $K$ of the open unit disk and an $\epsilon>0$, that we can find $c \neq 0$ and a polynomial $q$ such that $|h(z)-c q(z)|<\epsilon$, for $z \in K$.

Theorem 2.3. The set of strongly annular functions that satisfy equation (1.1) is dense in the set of all functions holomorphic on the open unit disk with the topology of almost uniform convergence.

Proof. It is enough to show that the set of polynomials can be approximated almost uniformly by such functions. Let $h(z)$ be a polynomial with complex coefficients, let $K$ be a compact subset of the open unit disk, and let $\epsilon>0$ be given. By Lemma 2.2 there is a polynomial $q$ satisfying (1.2) and a constant $c \neq 0$ so that $|h(z)-c q(z)|<\epsilon / 2$ on $K$.

Define the functions $L_{p}$ for each positive integer $p \geq 2$ as

$$
L_{p}(z)=\prod_{k=0}^{\infty} q\left(z^{p^{k}}\right) .
$$

It was shown by Daquila [5] that this product converges uniformly on compact subsets of the open unit disk. Hence, for each positive integer $p \geq 2, L_{p}$ is holomorphic in the open unit disk. It was also shown in [5] that $L_{p}$ satisfies (1.1) and is a strongly annular function. It is important to notice that as $p \rightarrow \infty$ the sequence of functions $L_{p}\left(z^{p}\right)$ converges to the function $a(z) \equiv 1$ uniformly on $K$.

Choose $p>\operatorname{deg} q(z)$ and large enough so that for all $z \in K$ we have a bound of $\left|c q(z)\left(1-L_{p}\left(z^{p}\right)\right)\right|<\epsilon / 2$. Then for $z \in K$ we have the estimate

$$
\begin{aligned}
\left|h(z)-c L_{p}(z)\right| & =\left|h(z)-c q(z) L_{p}\left(z^{p}\right)\right| \\
& \leq|h(z)-c q(z)|+\left|c q(z)\left(1-L_{p}\left(z^{p}\right)\right)\right| \\
& <\epsilon .
\end{aligned}
$$

Hence the strongly annular solutions of equation (1.1) are dense in the set of all holomorphic functions on the open unit disk with the topology of almost uniform convergence. 
Theorem 2.4. The set $M$ is a first category set in the topology of almost uniform convergence of all functions holomorphic on the open unit disk.

Proof. For each integer $p \geq 2$ and $n \geq 1$, define $M_{n, p}$ to be the collection of all solutions $g$ of the functional equation (1.1) such that $q$ is a polynomial of degree at most $n$. The set $M$ is then the countable union of the sets $M_{n, p}$. We will show that $M$ is of first category by showing that each $M_{n, p}$ is nowhere dense. To do this we will show that the closure of each $M_{n, p}$ contains no non-constant polynomials. As the non-constant polynomials are dense in the topology of almost uniform convergence this gives us that each $M_{n, p}$ is nowhere dense.

Suppose $g$ is a non-constant polynomial contained in the closure of $M_{n, p}$ for some $p \geq 2$ and $n \geq 1$. Let $g_{j}$ be a sequence of functions in $M_{n, p}$ that converges almost uniformly to $g$. It follows then that $g_{j}\left(z^{p}\right)$ converges almost uniformly to $g\left(z^{p}\right)$. Since $\frac{g(z)}{g\left(z^{p}\right)}$ is a polynomial of degree between 1 and $n$ for each $j \in \mathbb{N}$, we may conclude that $\frac{g_{j}(z)}{g_{j}\left(z^{p}\right)}$ is a polynomial of degree between 0 and $n$. Call this polynomial $q$. Note that $q \not \equiv 0$ since each polynomial $\frac{g_{j}(z)}{g_{j}\left(z^{p}\right)}$ takes on the value 1 at the origin. Therefore, we have $g(z)=q(z) g\left(z^{p}\right)$, which is a contradiction since the degrees of the polynomials on each side of this equation are different. In particular, $\operatorname{deg} g \neq \operatorname{deg} q+p \cdot \operatorname{deg} g$.

Combining Theorem 2.3 and Theorem 2.4 yields the main result of this section, which we now state.

Theorem 2.5. The subset of $M$ that consists of strongly annular functions is a dense first category subset of functions holomorphic on the unit disk with the topology of almost uniform convergence.

\section{Functions with Coefficients \pm 1}

The existence of polynomials with Maclaurin coefficients \pm 1 and whose minimum modulus is greater than 1 on $|z|=1$ has been studied by Carroll, Eustice and Figiel [6]. We use the following example. The polynomial function

$$
P(z)=1-z+z^{2}+z^{3}+z^{4}
$$

whose coefficients are all \pm 1 . If we make the substitution $z=e^{i \theta}$ and compute the square of the modulus we get the expression

$$
|P(z)|^{2}=\left|P\left(e^{i \theta}\right)\right|^{2}=5+2 \cos (2 \theta)+2 \cos (4 \theta) .
$$

One may check that the minimum value of this expression is $\sqrt{11} / 2$.

The first lemma of this section gives a construction of a sequence of polynomials with coefficients \pm 1 with various properties whose minimum modulus on the unit circle has a positive lower bound.

Lemma 3.1. Let $Q(z)$ be a polynomial of degree $m-1$ with coefficients \pm 1 and $Q(0)=1$. Define the sequence of polynomials $Q_{n}$ recursively by

$$
\begin{aligned}
Q_{1}(z) & =Q(z)=\sum_{j=0}^{m-1} \delta_{j} z^{j} \\
Q_{n+1}(z) & =Q_{n}\left(z^{m}\right) Q(z),
\end{aligned}
$$

where each $\delta_{j}= \pm 1$. The polynomials $Q_{n}$ satisfy each of the following conditions: 
(i) $Q_{n}(z)$ is of degree $m^{n}-1$.

(ii) $Q_{n}(z)$ has coefficients of \pm 1 .

(iii) $Q_{n}(z)=\prod_{\nu=0}^{n-1} Q\left(z^{m^{\nu}}\right)$.

(iv) $Q_{n}(z)-Q(z)$ has a zero of order $m$ at the origin for $n \geq 2$.

(v) If $\min _{|z|=1}|Q(z)|>\alpha$, then $\min _{|z|=1}\left|Q_{n}(z)\right| \geq \alpha^{n}$.

Proof. The proof is by induction where the case $n=1$ is obvious by the definition of $Q_{1}=Q$. Assume for the purpose of induction that

$$
Q_{n}(z)=\prod_{\nu=0}^{n-1} Q\left(z^{m^{\nu}}\right)=\sum_{\nu=0}^{\mu} \epsilon_{\nu} z^{\nu}
$$

where $\epsilon_{\nu}= \pm 1$ and the degree of $Q_{n}$ is $\mu=m^{n}-1$. We apply the definition of $Q_{n+1}$ and get the following expression:

$$
\begin{aligned}
Q_{n+1}(z) & =Q_{n}\left(z^{m}\right) Q(z)=Q(z) \prod_{\nu=0}^{n-1} Q\left(z^{m^{\nu+1}}\right)=\prod_{\nu=0}^{n} Q\left(z^{m^{\nu}}\right) \\
& =\sum_{\nu=0}^{\mu} \epsilon_{\nu} z^{m \nu} Q(z)=\sum_{\nu=0}^{\mu} \epsilon_{\nu} z^{m \nu} \sum_{j=0}^{m-1} \delta_{j} z^{j}=\sum_{\nu=0}^{\mu} \sum_{j=0}^{m-1} \epsilon_{\nu} \delta_{j} z^{m \nu+j}
\end{aligned}
$$

The degree of the polynomial $Q_{n+1}$ is $m^{n+1}-1$, which we obtain when $\nu=\mu=m^{n}-$ 1 and $j=m-1$ are substituted in the last term. Clearly the exponents $m \nu+j$ in the last written sum are distinct and assume all values in the set $\left\{0,1, \ldots, m^{n+1}-1\right\}$, so we can write

$$
Q_{n+1}(z)=\sum_{k=0}^{\mu_{0}} \gamma_{k} z^{k}
$$

where $\gamma_{k}=\epsilon_{\nu} \delta_{j}= \pm 1$ and $\mu_{0}=m^{n+1}-1$.

The order of the zero at the origin can be obtained by considering the definition of $Q_{n}$ :

$$
Q_{n+1}(z)-Q(z)=Q_{n}\left(z^{m}\right) Q(z)-Q(z)=Q(z)\left(Q_{n}\left(z^{m}\right)-1\right) .
$$

Since $Q(0)=1$ and all terms of the polynomial $Q_{n}\left(z^{m}\right)-1$ are of degree $m$ or higher, the order of the zero at the origin is $m$.

The bound on the minimum modulus in (v) follows easily from the expression in (iii).

Lemma 3.2. Given a polynomial $h(z)=\sum_{j=0}^{m_{0}} \epsilon_{j} z^{j}$ with coefficients $\epsilon_{j}= \pm 1$, there exists a polynomial $q(z)=\sum_{j=0}^{m} \delta_{j} z^{j}$, with coefficients $\delta_{j}= \pm 1, m>m_{0}, \delta_{j}=\epsilon_{j}$ for $0 \leq j \leq m_{0}$ and

$$
\min _{|z|=1}|q(z)|>1 \text {. }
$$

Proof. Consider the sequence of polynomials $Q_{n}$ constructed in Lemma 3.1 with $Q_{1}=P$ given by (3.1). Let $\alpha=\sqrt{11} / 2$ and choose a natural number $n_{0}$ large enough so that $\alpha^{n_{0}}>2\left(m_{0}+1\right)+1$. Consider the polynomial

$$
Q_{n_{0}}(z)=\sum_{k=0}^{m} \gamma_{k} z^{k}
$$


where $\gamma_{k}= \pm 1$ and $m=5^{n_{0}}-1$. We note that $m>m_{0}$. Define the set $\mathcal{K}$ of indices $0 \leq k \leq m_{0}$ such that $\gamma_{k} \neq \epsilon_{k}$. Because $\gamma_{k}$ and $\epsilon_{k}$ are either \pm 1 we have $\gamma_{k}=-\epsilon_{k}$ when $k \in \mathcal{K}$.

Define the polynomials $q_{0}$ and $q(z)$ by:

$$
\begin{aligned}
q_{0}(z) & =\sum_{k \in \mathcal{K}} \gamma_{k} z^{k} \\
q(z) & =Q_{n_{0}}(z)-2 q_{0}(z)=\sum_{k=0}^{m} \delta_{k} z^{k} .
\end{aligned}
$$

It remains to show that $\delta_{k} \in\{-1,1\}$ for $k=0,1, \ldots, m$, that $\delta_{k}=\epsilon_{k}$ for $k=0,1, \ldots, m_{0}$, and that $|q(z)|>1$ when $|z|=1$. For $k \notin \mathcal{K}$ we get $\delta_{k}=\gamma_{k}= \pm 1$ and for $k \in \mathcal{K}$ we have $\delta_{k}=-\gamma_{k}=\mp 1$; hence all coefficients are \pm 1 . For $0 \leq k \leq m_{0}$ we have $\delta_{k}=\gamma_{k}=\epsilon_{k}$ for $k \notin \mathcal{K}$ and $\delta_{k}=-\gamma_{k}=\epsilon_{k}$ for $k \in \mathcal{K}$. Consider $|z|=1$ in the estimate for $q$ below:

$$
\begin{aligned}
|q(z)| & =\left|Q_{n_{0}}(z)-2 q_{0}(z)\right| \\
& \geq\left|Q_{n_{0}}(z)\right|-\left|2 q_{0}(z)\right| \\
& \geq \alpha^{n_{0}}-2 \sum_{k \in \mathcal{K}}\left|\gamma_{k} z^{k}\right| \\
& >2\left(m_{0}+1\right)+1-\left(2\left(m_{0}+1\right)\right),
\end{aligned}
$$

since there are no more than $m_{0}+1$ terms in the sum for $q_{0}$. Hence the minimum modulus condition is satisfied.

We next state and prove the major result of this section.

Theorem 3.3. The set of strongly annular solutions of the functional equations given by (1.1) where $q(z)$ is a polynomial with coefficients of \pm 1 with $q(0)=1$ and the Maclaurin coefficients of $g(z)$ are all \pm 1 is a dense set in the set of all holomorphic functions in the open unit disk with Maclaurin coefficients of \pm 1 in the topology of almost uniform convergence.

Proof. Let $K$ be a compact set in the open unit disk, let $H(z)$ be a holomorphic function with Maclaurin coefficients of \pm 1 and let $\epsilon>0$ be given. Since $K$ is compact there exists $r$ with $0<r<1$ such that $|z|<r$ for all $z \in K$. Choose a natural number $N$ such that if $n>N$, then $2 r^{n} /(1-r)<\epsilon$. Let $n_{0}>N$ and let $h(z)$ be the Taylor polynomial of degree $n_{0}$ for $H(z)$ (i.e. the Maclaurin coefficients of $h$ and $H$ are equal for terms of degree $n_{0}$ or less).

For now let us assume that $h(0)=H(0)=1$. By Lemma 3.2 there exists a polynomial $q(z)$ with coefficients \pm 1 of degree $m>n_{0}$ whose minimum modulus on $|z|=1$ is greater than 1 , and all of the coefficients of $h$ are equal to the corresponding coefficients of $q$. Let $p=m+1$ and define the function $g$ as

$$
g(z)=\prod_{\nu=0}^{\infty} q\left(z^{p^{\nu}}\right)=\lim _{n \rightarrow \infty} \prod_{\nu=0}^{n} q\left(z^{p^{\nu}}\right) .
$$

The infinite product converges almost uniformly on compact subsets of the open unit disk and thus represents a holomorphic function. The function $g$ is a strongly annular solution to the functional equation (1.1) since $q$ satisfies conditions (1.2). It follows from Lemma 3.1 that the Maclaurin coefficients of $g$ are all \pm 1 and that 
all coefficients of $h$ agree with the corresponding coefficients of $g$. If $z \in K$, then $|z|<r$ and hence

$$
|H(z)-g(z)| \leq|H(z)-h(z)|+|h(z)-g(z)| \leq \frac{2 r^{n_{0}}}{1-r}<\epsilon .
$$

In the case that $h(0)=H(0)=-1$, replace $h$ by $-h$ and obtain the function $g$ as above. If $g$ is strongly annular and a solution to (1.1), then the function $-g$ will have both properties.

Hence the solutions of (1.1) are dense.

\section{REFERENCES}

1. P. Becker, Effective measures for algebraic independence values of Mahler type functions, Acta Arith. 58 (1991), no. 3, 239-250. MR.1121085 (92h:11061)

2. D.D. Bonar, On Annular Functions, Veb Deutscher Verlag Der Wissenschaften, Berlin, 1971. MR 0450560 (56:8853)

3. D.D. Bonar and F.W. Carroll, Annular functions form a residual set, J. Reine Agnew. Math. 272 (1975), 23-24. MR0417428 (54:5478)

4. J. Conway, Functions of One Complex Variable, 2nd ed., Springer-Verlag, New York, 1978. MR503901 (80c:30003)

5. R. Daquila, Strongly annular solutions of Mahler's functional equation, Complex Variables Theory Appl. 32 (1997), 99-104. MR1448483 (98c:30034)

6. D. Eustice, F.W. Carroll and T. Figiel, The minimum modulus of polynomials with coefficients of modulus one, J. London Math. Soc. (2) 16 (1977), no. 1, 76-82. MR.0480955 (58:1102)

7. R.W. Howell, Annular functions in probability, Proc. Amer. Math. Soc. 52 (1975), 217-221. MR0374398 (51:10598)

Department of Mathematics, Muskingum University, New Concord, Ohio 43762

E-mail address: rdaquila@muskingum.edu 